\title{
Polarization gratings and electro-optics of deformed helix ferroelectric liquid crystals
}

\author{
Alexei D. Kiselev, ${ }^{* 1,2}$ Eugene P. Pozhidaev, ${ }^{1,3}$ Vladimir G. Chigrinov, ${ }^{1}$ and Hoi-Sing Kwok ${ }^{1}$ \\ ${ }^{1}$ Hong Kong University of Science and Technology, Clear Water Bay, Kowloon, Hong Kong, \\ ${ }^{2}$ Institute of Physics of National Academy of Sciences of Ukraine, prospect Nauki 46, 03028, Kyiv, Ukraine, \\ ${ }^{3}$ P.N. Lebedev Physics Institute of Russian Academy of Sciences, Leninsky prospect 53, 117924, Moscow, Russia
}

Received February 15, 2011; accepted February 18, 2011; published March 31, 2011

\begin{abstract}
Electro-optical properties of deformed helix ferroelectric liquid crystal (DHFLC) cells are studied by using a general theoretical approach to polarization gratings in which the transmission and reflection matrices of diffraction orders are explicitly related to the evolution operator of equations for the Floquet harmonics. DHFLC cells with a subwavelength pitch are found to be optically equivalent to uniformly anisotropic biaxial layers. We calculate the transmittance as a function of the electric field and compare the results with the experimental data. The theoretical and experimental results are found to be in good agreement.
\end{abstract}

A polarization grating (PG) can generally be described as an optically anisotropic layer characterized by anisotropy parameters that periodically vary in space along a line in the plane of its input face. Unlike conventional phase and amplitude diffraction gratings, PGs act by locally modifying the polarization state of light waves passing through them. Owing to the onedimensional (1D) in-plane periodicity, this introduces periodically modulated changes of the polarization characteristics giving rise to polarization-dependent diffraction. In particular, the latter implies that a PG divides a monochromatic plane wave into differently polarized diffracted waves.

Over the past decade PGs have attracted much attention due to a unique combination of their optical properties: (a) it is possible to achieve $100 \%$ diffraction into a single order; (b) diffraction efficiencies are highly sensitive to incident light polarization; and (c) the state of polarization of diffracted orders is determined solely by the parameters of a PG [1-3]. There are numerous applications in a variety of fields, including polarimeters, displays, polarizing beam splitters, beam steering and polarization multiplexers where PGs have been found to be useful [4-6].

The well-known method to produce PGs is based on polarization holography [6]. It uses two differently polarized light beams to record a spatially modulated polarization state of the resultant light field on suitable media such as azobenzene containing polymer systems and silver-halide materials.

\footnotetext{
"E-mail: kiselev@iop.kiev.ua
}

The holographic technique has been extensively used to create polarization gratings in liquid crystal (LC) cells with photosensitive aligning substrates such as linear photopolymerizable polymer layers [7, 8], azo-dye films [9], azo-dye doped polyimide [10, 11], and azobenzene side-chain polymer layers [12].

In this method, irradiation of the substrate with a holographically generated polarization interference pattern gives rise to spatially modulated light induced ordering in the photoaligning layer. This ordering manifests itself in the effect of photoinduced optical anisotropy and determines the anchoring properties of the layer such as its (polar and azimuthal) anchoring strengths and easy axis orientation [13].

The anchoring parameters of the photoaligning film thus undergo periodic variations across the substrate face leading to the formation of orientational structures in the liquid crystal cells characterized by spatially periodic distributions of the liquid crystal director, $\mathbf{d}=\left(d_{x}, d_{y}, d_{z}\right)$, which is a unit vector that denotes a local direction of the preferential orientation of LC molecules. In liquid crystals, the elements of the dielectric tensor, $\varepsilon$, can be expressed in terms of the LC director

$$
\varepsilon_{i, j}=\varepsilon_{\perp}\left(\delta_{i, j}+u_{a} d_{i} d_{j}\right), \quad u_{a}=\left(\varepsilon_{\|}-\varepsilon_{\perp}\right) / \varepsilon_{\perp},
$$

where $\delta_{i, j}$ is the Kronecker symbol, $u_{a}$ is the anisotropy parameter and $n_{\perp} \equiv n_{o}=\sqrt{\mu \varepsilon_{\perp}} \quad\left(n_{\|} \equiv n_{e}=\sqrt{\mu \varepsilon_{\|}}\right)$is ordinary (extraordinary) refractive index (the magnetic tensor of LC is assumed to be isotropic with the magnetic permittivity $\mu$ ). So, the periodic orientational LC configurations define the so-called liquid crystal polarization gratings (LCPG). These gratings will be of our primary interest.

Common methods most generally employed to derive theoretical results for PGs typically rely on the wellknown Jones matrix formalism and its modifications [1-4, $6,12]$. These results are limited by their assumptions to large grating periods and normal incidence. In addition, using Jones calculus implies neglecting multiple reflections. 
In this work we follow the theoretical approach to PGs, which is derived as a generalization of our method developed in $[14,15]$ for stratified anisotropic media and goes beyond the limitations of the Jones calculus (details can be found in [16]).

Specifically, we deal with the light transmission problem for a polarization grating in the slab geometry. In this geometry, the $z$ axis is normal to the bounding surfaces of the layer: $z=0$ and $z=D$, the grating with the grating pitch, $\Lambda_{g}$, and the grating wave vector, $\mathbf{k}_{g}=k_{g} \mathbf{x}$, where $k_{g}=2 \pi / \Lambda_{g}$ is the grating wave number, is characterized by the condition of in-plane periodicity for the elements of the dielectric tensor, $\varepsilon$ : $\varepsilon_{i, j}\left(x+\Lambda_{g}\right)=\varepsilon_{i, j}(x)$. So, the $x-z$ plane is defined as the plane of grating.

We apply the method for a systematic treatment of the technologically important case of the deformed helix ferroelectric liquid crystals (DHFLCs) $[17,18]$ where the liquid crystal director

$$
\mathbf{d}=\left(d_{x}, d_{y}, d_{z}\right)=\left(\cos \theta_{t}, \sin \theta_{t} \cos \Phi, \sin \Phi\right)
$$

lies on the smectic cone with the smectic tilt angle $\theta_{t}$ and rotates in a helical fashion about a uniform in-plane twist axis (the $x$-axis) forming the ferroelectric LC (FLC) helical structure.

In the presence of weak electric field, $\mathbf{E}=E \mathbf{z}$, which is well below its critical value at the helix unwinding transition, $E<<E_{C}$, the azimuthal angle around the cone, $\Phi$, can be written in the form [18-20]

$$
\Phi=\varphi+\Delta \Phi(\varphi) \approx \varphi+\alpha_{E} \sin \varphi, \quad \varphi=\frac{2 \pi}{P} x,
$$

where the electric field induced distortion $\Delta \Phi$ linearly depend on $E$ through the electric field parameter $\alpha_{E}$ proportional to the ratio of the applied and critical electric fields: $E / E_{C}$. So, in the regime of weak electric field, the helix pitch $P$ is equal to the grating period, $\Lambda_{g}=P$.

In DHFLC cells, the FLC helix is characterized by a short submicron helix pitch, $P<1 \mu \mathrm{m}$, and a relatively large tilt angle, $\theta_{t}>30^{\circ}$. Electro-optical response of DHFLC cells exhibits a number of peculiarities that make them useful for LC devices such as high speed spatial light modulators [19, 21, 22] and colour-sequential liquid crystal display cells [20]. So, in this study, our goal is to examine electro-optical properties of DHF liquid crystals based on the general theoretical approach describing polarization gratings.

In our experiments, the transmittance of light passing through crossed polarizers was measured as a function of the applied electric field. We used the FLC mixture FLC-
576A (from P. N. Lebedev Physical Institute of Russian Academy of Sciences) as a material for the DHFLC layer. At room temperature, this mixture has the helix pitch, $P$, around $200 \mathrm{~nm}$.

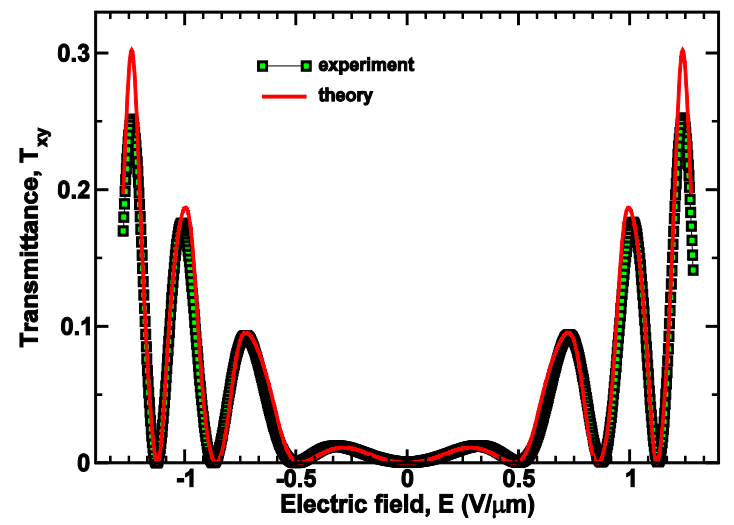

Fig. 1. Transmittance of light passing through crossed polarizers, $\mathbf{T}_{\mathrm{x}, \mathrm{y}}$ as a function of the applied electric field for the DHFLC cell of thickness $D=120 \mu \mathrm{m}$ filled with the FLC mixture FLC-576A. Solid line represents the theoretical curve computed for the electric field parameter $\alpha_{\mathrm{E}}=\gamma_{\mathrm{E}} \mathrm{E}$ with $\gamma_{\mathrm{E}} \approx 0.64 \mu \mathrm{m} / \mathrm{V}$.

The cells placed between crossed polarizers were illuminated with the light from a semiconductor Ga-As laser $(\lambda=650 \mathrm{~nm})$ and the output was collected by a photodetector.

The transmittance versus electric field curve shown in Fig. 1 presents the results measured in the cell in which the thickness of the DHFLC layer, $D$, was about $130 \mu \mathrm{m}$. Similarly, in Fig. 2, the transmittance $\mathbf{T}_{x y}$ is plotted against the applied electric field, $E$, for the case where the cell thickness is about $52 \mu \mathrm{m}$.

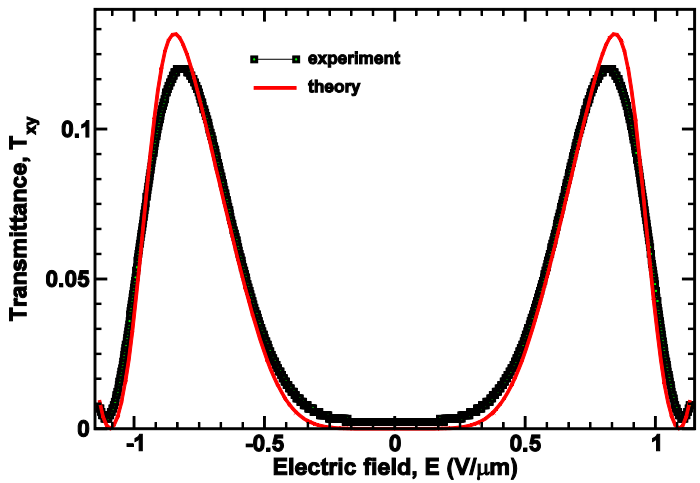

Fig. 2. Light transmittance, $\mathbf{T}_{\mathrm{x}, \mathrm{y}}$, of the DHFLC cell versus applied electric field, $E$. The cell thickness is estimated at about $D=52 \mu \mathrm{m}$. Solid line represents the theoretical curve.

In order to interpret the experimental data, theoretical analysis can be performed by using a short-pitch approximation which is applicable in the limiting case of chiral smectic helical configurations with the subwavelength helix pitch, $P<\lambda$. In this approximation, it 
can be shown that such DHFLC cells are optically equivalent to uniformly anisotropic biaxial layers. In these layers, the diagonal element

$$
\varepsilon_{\perp}^{-1} \varepsilon_{z z}^{(z z)}=n_{z}^{2} / n_{o}^{2}=\langle\eta\rangle^{-1}
$$

where $\quad \eta=1 /\left(1+v \sin ^{2} \Phi\right), \quad v=u_{a} \sin ^{2} \theta_{t} \quad$ and $\langle\ldots\rangle=(2 \pi)^{-1} \int_{0}^{2 \pi} \ldots d \varphi$, gives the principal value of the effective dielectric tensor for the optic axis normal to the cell (parallel to the $z$ axis), whereas in-plane anisotropy is characterized by the averaged dielectric tensor

$$
\varepsilon_{\perp}^{-1} \varepsilon_{\alpha \beta}^{(e f f)}=\delta_{\alpha \beta}+u_{a}\left\langle\eta d_{\alpha} d_{\beta}\right\rangle,
$$

where $\alpha, \beta \in\{x, y\}$.

Similar to the LC dielectric tensor (1), the tensor (5) can be expressed in terms of the in-plane optical axis, $\left(\cos \varphi_{d}, \sin \varphi_{d}, 0\right)$ with the azimuthal angle, $\varphi_{d}$, and its eigenvalues, $\varepsilon_{ \pm}=n_{ \pm}^{2}$. These parameters along with the thickness parameter $h=2 \pi D / \lambda$ determine the transmittance $\mathbf{T}_{x y}$ (the formula can be found in [16]) that describes the intensity of the light passing through crossed polarizers when the incident wave is linearly polarized along the helix axis and can be used to evaluate dependence of on the applied electric field, $E$. The known parameters characterizing the FLC mixture FLC-576A that enter our formulas are the ordinary (extraordinary) refractive index and the tilt angle estimated at $n_{o}=1.5$ $\left(n_{e}=1.72\right)$ and $\theta_{t}=32 \mathrm{deg}$, respectively. So, for the anisotropy parameters, $u_{a}$ and $v$, we have: $v \approx 0.315$ and $v=u_{a} \sin ^{2} \theta_{t} \approx 0.09$.

From the discussion after (3), the electric field parameter $\alpha_{E}$ is proportional to the electric field, $\alpha_{E}=\gamma_{E} E$, and thus is determined by the coefficient of proportionality $\gamma_{E}$. This coefficient is the sole fitting parameter in our calculations.

Figures 1 and 2 show that the theoretical curves computed at $\gamma_{E} \approx 0.64 \mu \mathrm{m} / \mathrm{V}$ and the experimental data are in close agreement. Figure 3 also demonstrates that the transmittance versus electric field curve will flatten in the vicinity of the origin when the cell thickness is chosen in such a way that the birefringence dependent factor equals zero at $E=0$.

In conclusion, we have used the method developed in [16] as a tool of theoretical investigation into the electrooptical properties of deformed helix ferroelectric liquid crystal gratings with a subwavelength pitch. We have shown that such DHFLC cells are equivalent to uniformly anisotropic biaxial layers. We have used the analytical results to evaluate the light transmittance measured in our experiments and found that the predictions of the theory are in good agreement with the experimental data.

This work is supported by HKUST grant CERG 612208, CERG 612310 and CERG 612409. A.D.K acknowledges partial financial support under STCU Grant No. 4687. E.P.P. was also supported by Russian Foundation of Basic Research (RFBR), Grants 09-0312234-ofi-m, 09-03-12263-ofi-m, 10-02-01336-a, 10-0313305-omi, 10-03-90016-Bel-a, 08-02-01074-a, by Russian Federal Agency of Science and Innovations (project 02.740.11.5166).

\section{References}

[1] T. Todorov, N. Tomova, L. Nikolova, Appl. Optics 23, 4309 (1984).

[2] F. Gori, Opt. Lett. 24, 584 (1999).

[3] J. Tervo, J. Turunen, Opt. Lett. 25, 785 (2000).

[4] G. Cincotti, IEEE Jour. Quant. Electr. 39, 1645 (2003)

[5] S.R. Nersisyan, N.V. Tabiryan, D.M. Steeves, B.R. Kimball, Jour. Nonlinear Opt. Phys. Mater. 18, 1 (2009).

[6] L. Nikolova, P.S. Ramanujam, Polarization Holography (Cambridge University Press, Cambridge, 2009).

[7] J.N. Eakin, Y. Xie, R.A. Pelcovits, M.D. Radcliffe, G.P. Crawford, Appl. Phys. Lett. 85, 1671 (2004).

[8] G.P. Crawford, J.N. Eakin, M.D. Radclie, A. Callan-Jones, R.A. Pelcovits, Jour. Appl. Phys. 98, 123102 (2005).

[9] V. Presnyakov, K. Asatryan, T. Galstian, V. Chigrinov, Opt. Exp. 14, 10558 (2006).

[10] C. Provenzano, P. Pagliusi, G. Cipparrone, Appl. Phys. Lett. 89, 121105 (2006).

[11] C. Provenzano, P. Pagliusi, G. Cipparrone, Opt. Exp. 15, 5872 (2007).

[12] H. Choi, J.W. Wu, Jour. Opt. Soc. Am. B 26, 1 (2009).

[13] A.D. Kiselev, V.G. Chigrinov, D.D. Huang, Phys. Rev. E 72, 061703 (2005).

[14] A.D. Kiselev, Jour. Phys.: Condens. Matter 19, 246102 (2007).

[15] A.D. Kiselev, R.G. Vovk, R.I. Egorov, V.G. Chigrinov, Phys. Rev. A 78, 033815 (2008).

[16] A.D. Kiselev, E.P. Pozhidaev, V.G. Chigrinov, H.-S. Kwok, arXiv:1011.4415

[17] L.A. Beresnev, V.G. Chigrinov, D.I. Dergachev, E.P. Poshidaev, J. Funfschilling, M. Schadt, Liq. Cryst. 5, 1171 (1989).

[18] V.G. Chigrinov, Liquid crystal devices: Physics and Applications (Artech House, Boston, 1999).

[19] I. Abdulhalim, G. Moddel, Mol. Cryst. Liq. Cryst. 200, 79 (1991).

[20] G. Hedge, P. Xu, E. Pozhidaev, V. Chigrinov, H.S. Kwok, Liq. Cryst. 35, 1137 (2008).

[21] E. Pozhidaev, S. Pikin, D. Ganzke, S. Shevtchenko, W. Haase, Ferroelectrics 246, 1141 (2000).

[22] G.B. Cohen, R. Pogreb, K. Vinokur, D. Davidov, Appl. Optics 3, 455 (1997). 